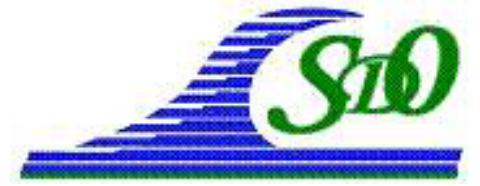

\title{
Suivi du trait de côte en Aquitaine par imagerie Formosat-2
}

\author{
Virginie LAFON ${ }^{1}$, Anaïs HOAREAU ${ }^{2}$, \\ Cyril MALLET ${ }^{2}$, Jean-François DESPRATS ${ }^{3}$
}

1. G.E.O. Transfert, UMR CNRS 5805 EPOC, Université Bordeaux 1, Avenue des Facultés, 33405 Talence cedex, France.

v.lafon@epoc.u-bordeaux1.fr

2. BRGM Aquitaine, 24, avenue Léonard de Vinci, 33600 Pessac, France. a.hoareau@brgm.fr ; c.mallet@brgm.fr

3. BRGM, Service Risques Naturels, 1039, rue de Pinville, 34000 Montpellier, France. jf.desprats@brgm.fr

\section{Résumé :}

Parmi ses missions de protection et de surveillance du littoral atlantique, l'Observatoire de la Côte Aquitaine assure le suivi du trait de côte en Aquitaine à l'aide d'observations DGPS (40 transects répartis sur les $240 \mathrm{~km}$ de plage sableuse).

Afin de compléter les observations in situ, et de produire une carte complète des faciès sédimentaires à l'échelle de l'ensemble de la côte sableuse aquitaine, le potentiel de l'imagerie satellite haute résolution Formosat-2 a été testé. Trois jeux successifs de 14 scènes multispectrales ont été acquis en août 2007, mai 2008 et mai 2009.

Une approche par classification de pixels a été définie, laquelle appliquée à des mosaïques Formosat permet de restituer des cartes thématiques des faciès littoraux. Le trait de côte (défini comme le pied de dune) est déduit de l'analyse des cartes doublée de l'analyse des scènes Formosat filtrées à l'aide de l'algorithme de Sobel.

Cette approche a été validée à l'aide de plus de 1300 mesures DGPS in situ. La précision globale des cartes produites ("overall accuracy") est de $73 \%$ pour 5 classes ("Forêt", "Dune grise", "Dune blanche", "Haut de plage" et "Plage intertidale") et 2 limites géomorphologiques ("Forest/Dune" et "Dune/Plage").

Les évolutions ont été analysées à l'échelle du littoral aquitain en se basant sur l'imagerie spatiale Formosat complétée de données plus anciennes. A l'exception de quelques secteurs montrant des phases d'accrétion ou d'érosion prononcées $(>45 \mathrm{~m}$ d'amplitude), le déplacement du trait de côte reste globalement limité.

Le potentiel et les limites de la télédétection pour le suivi du trait de côte à l'échelle de la côte sableuse en Aquitaine sont détaillés dans ce manuscrit afin de définir un cadre prospectif précis pour le suivi du littoral sableux par un outil de suivi opérationnel.

Mots-clés :

Télédétection - Cartographie - Trait de côte - Gestion - Prévention de l'aléa érosion 


\section{Introduction}

L'Observatoire de la Côte Aquitaine est un outil d'aide à la décision pour la gestion et l'aménagement du littoral aquitain. Il est financé par l'Europe (FEDER), l'Etat, la Région Aquitaine, les Conseils Généraux de la Gironde, des Landes et des Pyrénées Atlantiques, le Syndicat Mixte du Bassin d'Arcachon, le BRGM et l'ONF.

L'un des objectifs de l'Observatoire de la Côte Aquitaine est le suivi régulier et fréquent de l'évolution géomorphologique du littoral dans le but de mettre à disposition des gestionnaires du littoral aquitain un outil d'aide à la décision, en particulier concernant la problématique de l'érosion côtière.

La côte sableuse de l'Aquitaine s'étend sur près de $240 \mathrm{~km}$ entre l'estuaire de la Gironde et l'embouchure de l'Adour. Sous l'action de la houle et des marées (mésotidales), cette côte est soumise à une érosion moyenne de 1 à $3 \mathrm{~m} / \mathrm{an}$, qui varie selon les périodes et les secteurs (amplitude plus forte au nord de la Gironde que dans les Landes, certaines zones pouvant être stables plusieurs années successives). Depuis 2007, deux approches complémentaires ont été retenues par l'Observatoire pour le suivi de ce littoral sableux : une cartographie in situ des indicateurs géomorphologiques du littoral basée sur le suivi de transects dune-plage levés tous les $6 \mathrm{~km}$ approximativement et une spatialisation de cette information à l'échelle du littoral à partir de cartes extraites de l'imagerie satellite Formosat-2.

La présente étude porte sur la détermination par télédétection des faciès géomorphologiques du système plage-dune. La méthode de cartographie des faciès et sa validation à partir de la base de données in situ seront détaillées. La méthode et les résultats de la comparaison entre les trois traits de côte successifs $(2007,2008,2009)$ et des mesures plus anciennes sera également présentée.

\section{Données}

\subsection{Imagerie Formosat-2}

Trois jeux successifs d'acquisitions Formosat-2 ont été utilisés pour cette étude. Les scènes multispectrales Formosat sont constituées de quatre canaux B1 $(0,45-0,52 \mu \mathrm{m}$,

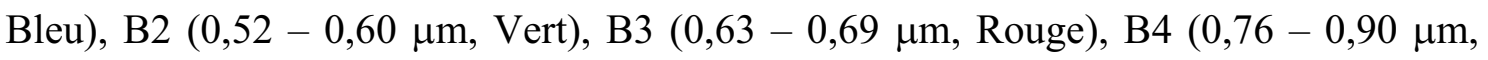
Proche Infra Rouge) et d'un canal panchromatique ( $\mathrm{P}: 0,45-0,90 \mu \mathrm{m})$. Les quatre premières bandes ont une résolution spatiale de $8 \mathrm{~m}$ et le canal panchromatique de $2 \mathrm{~m}$. Ces données ont été fusionnées afin de restituer les 4 bandes couleurs avec une résolution de $2 \mathrm{~m}$. Ces données ont été livrées par la société SpotImage, orthorectifiées, géoréférencées et en projection Lambert-2 Etendu / WGS 84. Chaque jeu de données est constitué de 14 scènes. En 2007, elles ont été acquises les 26 et 27 août, puis, en 2008, les 20 et 21 mai, et enfin, en 2009, les 20 et 28 mai. La programmation des images est faite afin que l'acquisition corresponde à une marée basse, à fort coefficient. 
La précision du positionnement des pixels FORMOSAT-2 a été évaluée à l'aide de mesures DGPS du contour d'un blockhaus. La précision observée est de l'ordre du pixel (2 m).

\subsection{Observations in situ}

Les observations de terrain sont organisées suivant 40 transects (forêt-plage) répétés régulièrement le long du littoral sableux de la côte aquitaine (près de $240 \mathrm{~km}$ au total) et également par la cartographie sur 3 secteurs (nord et sud de la Gironde et centre des Landes) des différentes limites des faciès géomophologiques du système plage-dune (pied de dune, limites dune-blanche-grise-forêt, ...). Au total, 1306 observations DGPS ont été utilisées pour valider les cartes spatiales. Chaque pointage porte un commentaire précis qui concerne le faciès rencontré, en particulier, la forêt de pin, la dune grise, la dune blanche, l'avant-dune et la dune embryonnaire, le trait de côte (qui souligne le pied de dune) le haut de plage et la plage intertidale. La faisabilité de détecter et de cartographier les faciès d'argiles, surtout visibles au nord de la côte aquitaine, et de landes, qui apparaissent au sud, a également été testée. Si la précision des mesures DGPS est centimétrique en $\mathrm{x}, \mathrm{y}, \mathrm{z}$, en revanche la localisation exacte des différents faciès géomorphologiques dépend de l'opérateur et des conditions naturelles et peut être estimée à $+/-5 \mathrm{~m}$ en moyenne.

\section{Méthode}

Plusieurs études successives ont permis de mettre au point une méthode d'extraction des faciès géomorphologiques sur le littoral aquitain (PEDREROS et al., 2005 ; LAFOSSE, 2007; MALLET et al., 2007). Cette méthode a été validée à l'aide d'un jeu d'observations DGPS exceptionnel levé en 2008 (MALLET et al., 2008).

La méthode d'analyse d'image comprend quatre étapes :

a) la constitution de mosaïques d'images présentant des caractéristiques sédimentologiques et des conditions atmosphériques homogènes ;

b) la définition des parcelles d'entraînement représentatives de la diversité des faciès par classification non supervisée (recuit simulé) de petits extraits des scènes satellite, nous cherchons à discriminer en priorité les indicateurs suivants : la forêt de pin, la dune grise, la dune blanche (avec laquelle l'avant-dune et la dune embryonnaire sont fusionnées), le haut de plage et la plage intertidale, les affleurements d'argile et les landes. La limite entre la forêt et la dune, ainsi que le pied de dune sont mises en évidence ;

c) la classification supervisée des scènes à partir des parcelles d'entraînement ;

d) la délinéation du trait de côte par une méthode de filtrage (algorithme de Sobel).

Les traits de côte 2007, 2008 et 2009 ont donc été doublement déterminés, par les cartes de faciès et les contours de Sobel. Les cartes extraites de l'imagerie Formosat-2 ont été validées en suivant l'approche décrite par FOODY (2002). 
A ce stade de l'analyse, l'expertise reste une étape inévitable pour vérifier que les traits de côte produits sont correctement positionnés par rapport aux différentes mesures de terrain, à l'intérieur de la zone d'incertitude.

L'étude de l'évolution des trois traits de côte est ensuite réalisée grâce à l'outil DSAS (Digital Shoreline Analysis System). Cet outil, logiciel libre développé par l'USGS, fonctionne sous ArcGIS CESRI (THIELER et al., 2009). Il permet de calculer les statistiques d'évolution d'une série de traits de côte. L'exécution de cette analyse nécessite au préalable la préparation des traits de côte au format vecteur au sein d'une géodatabase (une entité par année) et la numérisation d'une ligne de base (onshore ou offshore) à partir de laquelle DSAS créera des transects perpendiculaires qui viendront couper les différents traits de côtes. C'est à partir de ces points d'intersection que DSAS calcule le taux d'évolution entre les différents traits de côte. Il connaît en effet le temps écoulé entre deux traits de côte (date) et la distance entre les deux lignes (distance calculée entre les deux points d'intersection). En sortie, DSAS fournit une table contenant les statistiques d'évolution (taux de régression linéaire, moyenne des moindres carrés,...) et une table contenant les caractéristiques des points d'intersection (coordonnées, distance par rapport à la ligne de base).

\section{Résultats et discussion}

\subsection{Validation des cartes spatiales}

La validation porte sur le jeu de données spatiales obtenues à partir des scènes Formosat-2 acquises en 2008. Quatre mosaïques ont été constituées, à partir desquelles quatre cartes ont été générées. Ces cartes ont été croisées avec 1306 observations DGPS. La matrice de confusion pour 694 observations correspondant à 7 faciès est détaillée dans le tableau 1. A ces points de validation s'ajoutent 227 points DGPS qui marquent le trait de côte et 385 pour la limite de la forêt. Pour le trait de côte, l'interface entre les faciès de dune blanche et du haut de plage a été contrôlée à l'aide de l'image de Sobel qui met en évidence cette limite. Cette double détermination permet de valider la détection de la position du trait de côte à plus de $81 \%$. La limite de la forêt, détectée après classification des scènes Formosat, est validée à plus de $70 \%$.

En ce qui concerne les faciès, la procédure donne de bons résultats ( $>70 \%)$ pour la forêt, la dune grise, le haut de plage et l'argile. Le faciès de landes et la dune blanche approchent un score de 70\% (recommandé par THOMLINSON et al., 1999). Seule la plage intertidale est mal cartographiée, elle est fréquemment confondue avec le haut de plage. Toutefois, la distinction entre le haut de plage et la plage intertidale dépend de la laisse de mer qui était peut être différente au moment des prises de vue par rapport aux missions de terrain.

$\mathrm{Du}$ point de vue de l'utilisateur, ce sont le haut de plage et l'argile qui, seuls, apparaissent avec un score inférieur à $70 \%$, le haut de plage, parce qu'il est trop souvent 
confondu avec la plage intertidale, pour les raisons précédemment exposées. Les affleurements d'argile se situent sur la plage et sont incorrectement cartographiés et/ou discriminés du sable sombre et très humide rencontré sur la plage intertidale.

Tableau 1. Matrice de confusion ou matrice d'erreur de la classification.

\begin{tabular}{|c|c|c|c|c|c|c|c|c|c|c|}
\hline \multicolumn{11}{|c|}{ Observations } \\
\hline \multirow{10}{*}{$\begin{array}{l}\text { Pixels } \\
\text { classés }\end{array}$} & & $\boldsymbol{F}$ & $L$ & $D G$ & $D B$ & $H P$ & $A$ & $B P$ & Total & $\begin{array}{l}\text { \% Précision } \\
\text { utilisateur }\end{array}$ \\
\hline & Forêt $(F)$ & 22 & - & 7 & 1 & - & - & - & 30 & 73 \\
\hline & Landes $(L)$ & - & 9 & & - & - & - & - & 9 & 100 \\
\hline & Dune grise (DG) & 1 & 4 & 129 & 34 & - & - & 2 & 170 & 76 \\
\hline & Dune Blanche(DB) & 1 & - & 11 & 183 & 4 & - & 3 & 202 & 91 \\
\hline & Haut de plage (HP) & - & - & 5 & 48 & 70 & - & 49 & 172 & 41 \\
\hline & Argile (A) & - & - & - & - & - & 4 & 13 & 17 & 24 \\
\hline & Plage intertidale (PI) & - & - & - & 6 & 8 & - & 80 & 94 & 85 \\
\hline & Total & 24 & 13 & 152 & 272 & 82 & 4 & 147 & 694 & \\
\hline & \% Précision Procédure & 92 & 69 & 84 & 67 & 85 & 100 & 54 & & \\
\hline
\end{tabular}

La précision globale de la classification est en moyenne de 73,1\% (distinction des faciès "forêt", "dune grise", "dune blanche" (qui englobe l'avant dune et la dune embryonnaire), "haut plage", "plage intertidale", le trait de côte et la limite de la forêt). En regroupant ces faciès en trois classes majeures "Plage" ("plage intertidale" + "argile" + "haut de plage"), "Dune" ("dune grise" + "dune blanche" + "avant-dune") et en tenant compte de la détection de la limite de la forêt et du trait de côte, les scores obtenus durant la phase de validation passent de $73,1 \%$ à $81,5 \%$.

\subsection{Evolution récente du trait de côte du littoral sableux en Aquitaine}

L'analyse de l'évolution du trait de côte sableux aquitain est actualisée à partir des images Formosat. Les données plus anciennes ont également été utilisées (ex. : 1966, 1984, 1998, 2000, ...) et injectées dans l'outil DSAS afin de quantifier l'évolution du trait de côte en Aquitaine sur un plus long terme (figure 1). Cette étude confirme les tendances connues avec des taux d'évolution du pied de dune compris entre 0 et $1 \mathrm{~m} / \mathrm{an}$, pouvant atteindre dans des secteurs en érosion chronique plus de $4 \mathrm{~m} / \mathrm{an}$.

L'analyse régionale de ces tendances est encore en cours et fera l'objet d'un rendu fin 2010. Les analyses issues de DSAS, en particulier les évolutions déduites des positions successives du trait de côte, seront utilisées pour "projeter" les tendances déduites à 10 ou 20 ans en appliquant les taux calculés par DSAS aux points du trait de côte de référence. Cette cartographie se fait sous ArcGIS et Excel, DSAS ne permettant pas de dessiner ce nouveau trait. 


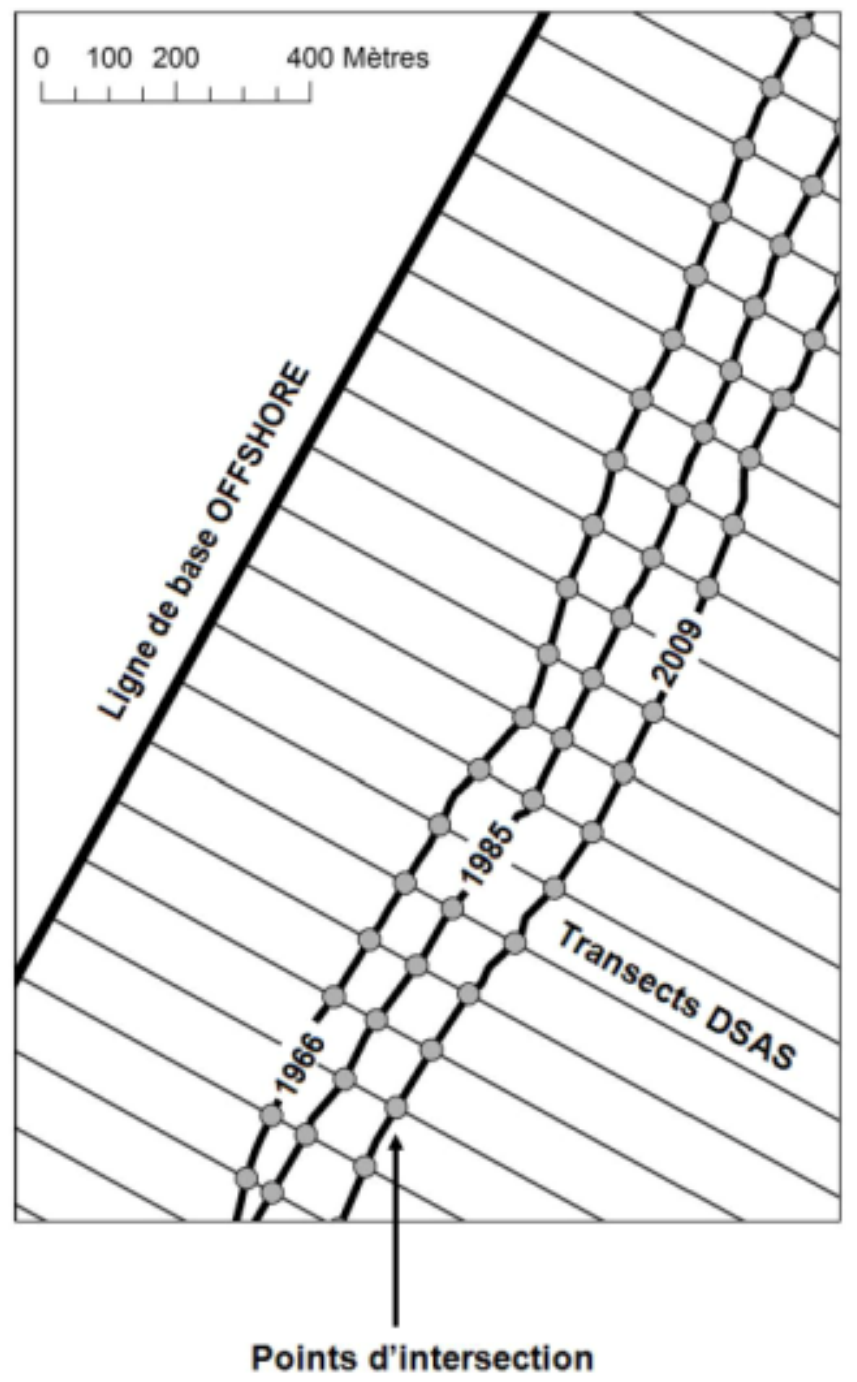

Figure 1. Analyse de l'évolution du trait de côte sur une portion du littoral sableux aquitain à l'aide de l'outil DSAS. Un trait de côte virtuel est tracé en mer (à gauche), il sert de base au tracé de transects qui lui sont perpendiculaires et recoupe les traits de côte mesuré sur le terrain ou par satellite en points d'intersection dont la position permet de calculer des statistiques concernant leur évolution spatiale.

\subsection{Limite de l'approche cartographique par télédétection}

Du point de vue de la cartographie thématique, nous avons vu qu'il était possible de discriminer différents faciès de dune et de plage. Toutefois en dégradant l'information cartographique (en regroupant les faciès en classes génériques, "forêt", "dune" et "plage", nous améliorons considérablement la validité de la carte Formosat-2. Cette perte d'information démontre le manque de connaissance sur certains faciès et notamment les faciès végétalisés. Leur discrimination sur l'image sera améliorée par leur observation directe sur le terrain. Toutefois, il faudrait acquérir des spectres de terrain sur les différents types de sable de la dune grise et de la dune blanche et des 
espèces végétales qui s'y développent afin de définir les faciès que l'on peut réellement discriminer à partir la résolution radiométrique réduite que permettent les capteurs spatiaux. Cette approche permettrait de définir la limite, faciès par faciès, de la méthode de cartographie décrite dans cette étude. D'autre part, une étude radiométrique doit être entreprise sur le terrain pour restituer plus finement la position des affleurements d'argile.

En ce qui concerne le trait de côte, la précision de son positionnement est limitée par plusieurs facteurs : la taille du pixel sur l'image tout d'abord, ainsi que la précision de la cartographie (10 $\mathrm{m}$ au total), et la précision du positionnement des levés DGPS (+/$5 \mathrm{~m}$ ). Les variations annuelles du pied de dune sont comprises entre 1 et $3 \mathrm{~m} / \mathrm{an}$ en moyenne. Il apparaît donc délicat de proposer des analyses d'évolution à un pas de temps annuel lorsque celle-ci est inférieure à $5 \mathrm{~m}$. En revanche cette approche est plus intéressante lorsque l'on compare des données temporellement plus espacées.

\section{Conclusions}

Cette étude montre l'intérêt de l'utilisation de l'imagerie spatiale pour un suivi régulier des indicateurs littoraux de la côte sableuse aquitaine, et en particulier de trois faciès majeurs (la forêt, la dune et la plage) et de deux limites géomorphologiques (la limite de la forêt et le trait de côte). Une approche de cartographie thématique a été développée pour cartographier les faciès littoraux, doublée d'un seuillage pour affiner la détection et le positionnement du trait de côte. Cette approche est fiable à plus de $80 \%$ pour la détection de ces trois classes et 2 limites, comme le montre la comparaison entre la carte satellite et les DGPS in situ (1306 points de contrôle). Cette précision est dégradée si plus de faciès sont pris en considération pour la classification. La détection de faciès plus nombreux doit être encore améliorée pour mieux restituer en particulier les faciès intra-dunaires, et notamment la végétation de la dune.

Les traits de côte Formosat complètent les observations plus anciennes et permettent de mettre à jour l'analyse des tendances actuelles d'évolution du littoral sableux. Une analyse exécutée avec le logiciel DSAS démontre des taux d'évolution du pied de dune compris entre 0 et $1 \mathrm{~m} / \mathrm{an}$ et pouvant dépasser $4 \mathrm{~m} / \mathrm{an}$ dans des secteurs en érosion chronique.

A l'avenir, l'acquisition régulière d'images soutiendra l'effort de l'Observatoire de la Côte Aquitaine pour ses missions d'expertise scientifique et technique d'aide à la décision, à la gestion et à la prévention des aléas de ce milieu en constante évolution.

\section{Références bibliographiques}

FOODY G.M. (2002). Status of land cover classification accuracy assessment. Remote Sensing of Environment, vol. 80, pp 185-201. doi:10.1016/S0034-4257(01)00295-4 
Thème 3 - Instrumentation, mesures, imagerie et télédétection

LAFOSSE M. (2007). Méthode de cartographie par télédétection des faciès morphosédimentaires du littoral aquitain. Master 2, BRGM/Université Bordeaux 1, $50 \mathrm{p}$.

MALLET C., LAFON V., DESPRATS J.F. (2007). Cartographie des faciès géomorphologiques du littoral aquitain à partir des données Formosat-2. BRGM/RP56101-FR. Rapport final $69 \mathrm{p}$.

MALLET C., LAFON V., DESPRATS J.F. (2008). Cartographie et évolution du trait de côte aquitain et analyse des faciès géomorphologiques à partir de données Formosat-2. Rapport BRGM.

PEDREROS R., DUPUIS H., MARIEU V., MALLET C., BUTEL R., OLIVEROS C., FROIDEFOND J.-M., HOSFORD S. (2005). Evaluation du potentiel de l'imagerie SPOT5 dans le secteur du Bassin d'Arcachon. BRGM/RP-54004-FR, 54 p.

THIELER E.R., HIMMELSTOSS E.A., ZICHICHI J.L., ERGUL A. (2009). Digital Shoreline Analysis System (DSAS) version 4.0-An ArcGIS extension for calculating shoreline change. U.S. Geological Survey Open-File Report 2008-1278.

THOMLINSON J.R., BOLSTAD P.V., COHEN W.B. (1999). Coordinating methodologies for scaling landcover classifications from site-specific to global: steps toward validating global map products. Remote Sensing of Environment, vol. 70, pp 16-28. doi:10.1016/\$0034-4257(99)00055-3 